\title{
The Effect of 4,4'-Methylenedianiline on Adrenal Corticosterone Secretion in the Rat
}

\author{
Yoshifumi Harada \\ Department of Physiology (Prof. T. Suzuki), \\ Nagasaki University School of Medicine, Nagasaki
}

\begin{abstract}
The effect of $4,4^{\prime}$-methylenedianiline on the adrenocortical secretion in the rat was investigated. Under sodium pentobarbital anesthesia, adrenal venous blood was collected through a polyethylene cannula inserted into the adrenal vein and analyzed for corticosterone. 4,4'-Methylenedianiline dihydrochloride was administered intravenously in a dose of $100 \mathrm{mg} / \mathrm{kg}$. In non-hypophysectomized rats, the increased corticosterone secretion in response to surgical stress caused by the adrenal vein cannulation was markedly depressed following the injection of methylenedianiline. A low secretion rate of corticosterone persisted for 30 minutes thereafter. In hypophysectomized rats, the increased corticosterone secretion produced by intravenous infusion of ACTH was also markedly depressed by injection of methylenedianiline. It is thus concluded that in the rat the adrenal corticosterone secretion is depressed by $\mathbf{4 , 4} \mathbf{4}^{\prime}$. methylenedianiline and that this inhibitory effect on adrenal corticosterone secretion is due to a direct action of the drug on the adrenal cortex.
\end{abstract}

The depressant effect of amphenone on the biosynthesis and secretion of corticosteroid of the adrenal gland is well established. ${ }^{1-6}$ Tullner $^{7}$ reported that the intravenous administration of methylenedianiline, a diaminodiphenyl compound analogous to amphenone $B$ in chemical structure, in a dose of 50 to $100 \mathrm{mg} / \mathrm{kg}$ of body weight, produced a marked decrease in the 17-hydroxycorticoid secretion rate in intact dogs. He also noted that the adrenal hypertrophy following oral administration of methylenedianiline was induced in rats. However, the effect of methylenedianiline on the adrenal cortical secretion of the rat has not directly been evaluated.

The present study was undertaken to ascertain whether the adrenal corticosterone secretion in rats is suppressed by administration of methylenedianiline as observed in 17-hydroxycorticoid secretion in dogs, and to clarify the problem whether the effect of methylenedianiline is manifested by its direct action on the adrenal gland or by indirect one mediated through the hypothalamo-pituitary system.

Received for publication, November 16, 1966. 


\section{METHODS}

Thirty male rats of Wistar strain, weighing 183 to $421 \mathrm{~g}$, were used in this study. Two series of experiments were performed.

In the first series of experiments, animals were anesthetized by intraperitoneal administration of sodium pentobarbital (Abbott) in a dose of $50 \mathrm{mg} / \mathrm{kg}$ of body weight. A small polyethylene tube was inserted into the left femoral vein for infusion of heparin-Ringer's solution. In order to collect the adrenal venous blood, the left adrenal gland was exposed through a pararectal abdominal incision and the left renal vein was ligated at two sites, near the kidney and near the inferior vena cava. The inferior phrenic vein was ligated too. A small polyethylene cannula was inserted through the renal vein into the adrenal vein. Immediately after completion of the adrenal vein cannulation, infusion of heparin-Ringer's solution and adrenal venous blood sampling were started. Adrenal venous blood samples were taken during six consecutive periods of 10 minutes' collection. Aqueous solution of methylenedianiline dihydrochloride (100 $\mathrm{mg}$ in $1 \mathrm{ml} \mathrm{of} 0.9 \%$ saline solution) was administered via the right femoral vein in a dose of $100 \mathrm{mg}$ per $\mathrm{kg}$ of body weight after collecting two samples. In control experiments the same volume of $0.9 \%$ saline solution was administered.

In the second series of experiments, hypophysectomy was performed under ether anesthesia through a parapharyngeal approach, completeness of hypophysectomy being checked at autopsy after the completion of experiments. Immediately after removal of the hypophysis, the animals were anesthetized with sodium pentobarbital in $50 \mathrm{mg} / \mathrm{kg}$ of body weight and the adrenal vein cannulation was also performed, as described above. One hour after the hypophysectomy, adrenal venous blood collection was begun. Sixty $\mathrm{mU}$ of ACTH (Biochemical Research Co.) was dissolved in $50 \mathrm{ml}$ of Ringer's solution and infused over 60 minutes at a rate of $0.396 \mathrm{mU}$ per minute. After collecting three samples, methylenedianiline was administered.

In both series of experiments, rectal temperature was measured. Electric heater was used to prevent a fall of body temperature. The adrenal venous blood was centrifuged immediately after collection and 0.1 to $1.0 \mathrm{ml}$ of plasma was analyzed for corticosterone by the fluorometric technique of Silber et al. ${ }^{8}$

\section{Results}

Results are shown in Tables 1 and 2. In as much as the pre-injection rates of corticosterone secretion were variable from rat to rat, the secretion rates in the 10 minutes' collection period just before the injection of methylenedianiline are taken in each rat as 100 per cent and all the secretion rates are expressed in the tables in percentage of the pre-injection rate in each animal. 


\section{1) Effect of methylenedianiline in the non-hypophysectomized rat}

In 7 non-hypophysectomized rats, saline solution was injected instead of methylenedianiline. The levels of corticosterone secretion rate were high as a result of surgical stress. Even after the injection of saline solution, no significant alteration in the corticosterone secretion rate was observed. It should be noted that significant changes were not observed in adrenal venous blood flow during experiments, since blood loss due to adrenal venous blood collection was replaced by continuous infusion of heparin-Ringer's solution.

TABLE 1. Effect of $4,4^{\prime}$-methylenedianiline on adrenal corticosterone secretion rate in non-hypophysectomized rats

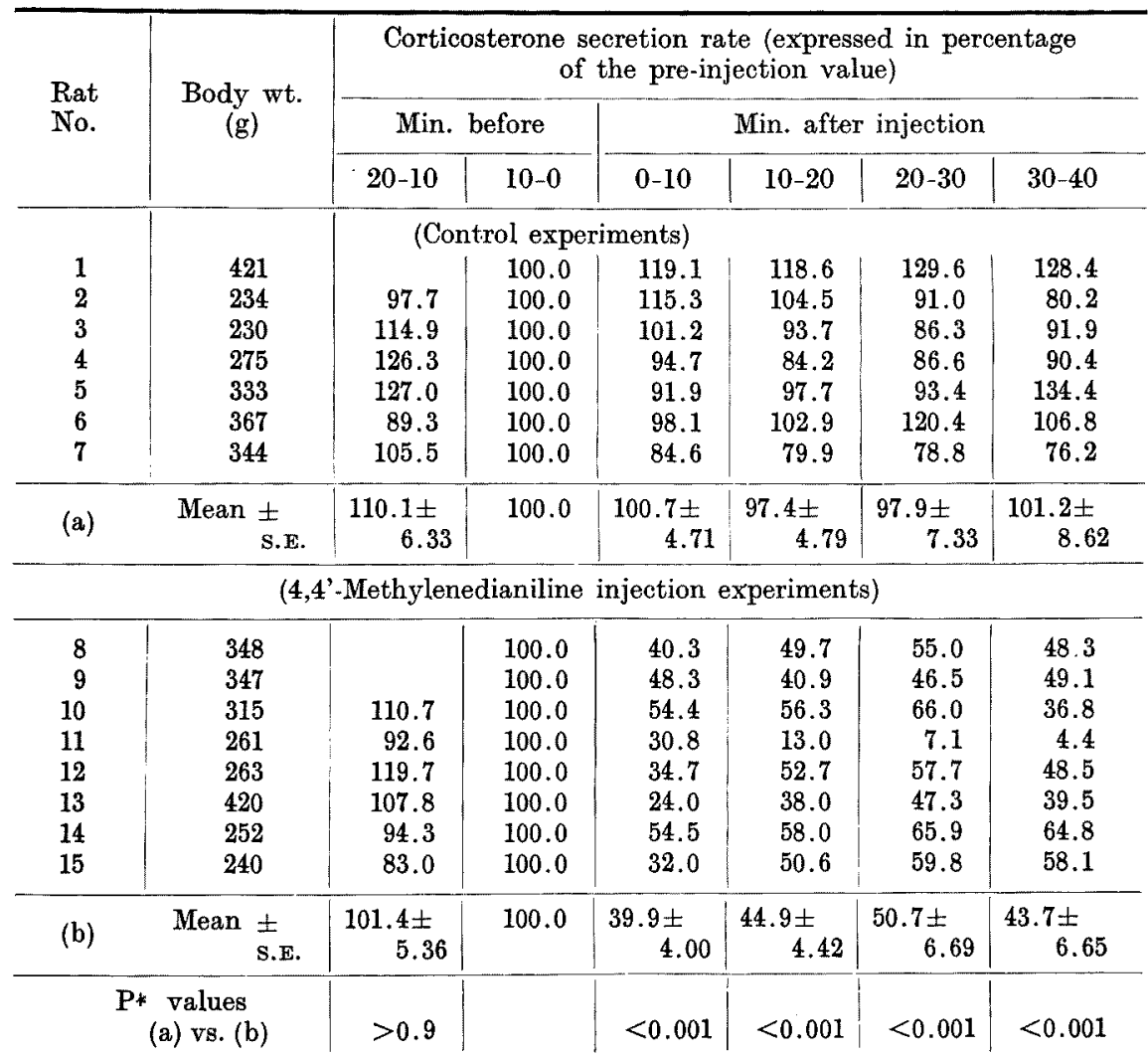

* Probability in statistical analysis by Student's $t$-test

Eight non-hypophysectomized rats were utilized for testing the effect of acute intravenous administration of methylenedianiline in a dose of $100 \mathrm{mg} / \mathrm{kg}$ of body weight. Immediately after the injection of methylenedianiline, the corticosterone secretion was markedly decreased and the depressant effect was 

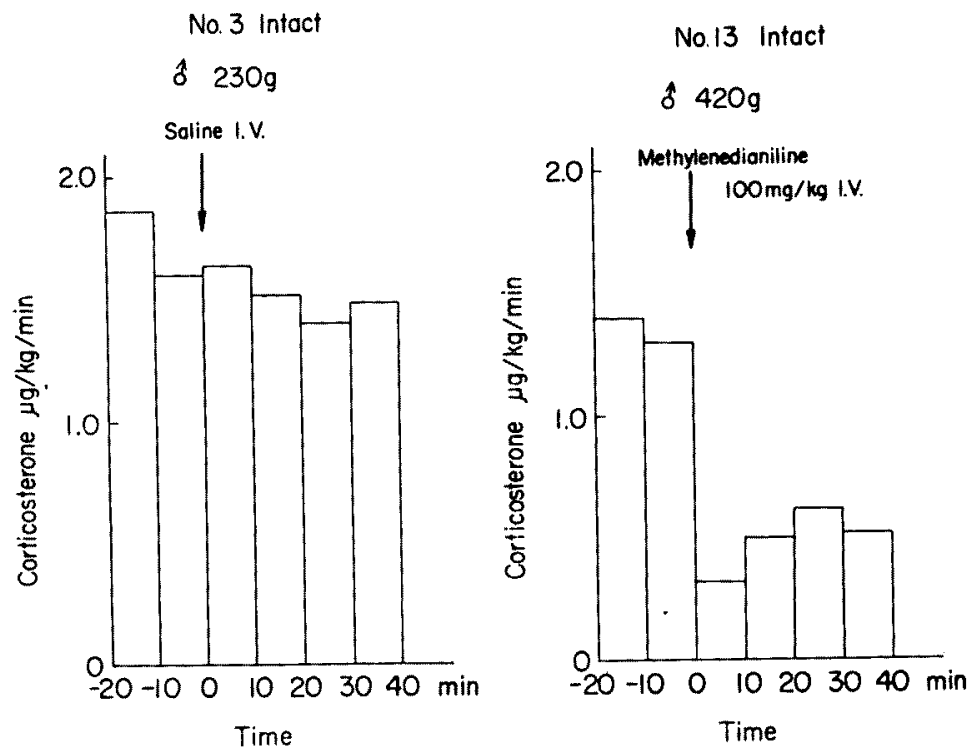

Fig. 1. Effect of 4,4'-methylenedianiline on adrenal corticosterone secretion rate in intact rats.

sustained for $40 \mathrm{~min}$. After the administration of methylenedianiline, adrenal blood flow tended to decrease. Respiratory rate was distinctly increased immediately after the beginning of the administration and the increase continued for a few minutes.

Fig. 1 shows a change of adrenal corticosterone secretion rate in rat 3 (control) and rat 13 (methylenedianiline administered).

\section{2) Effect of methylenedianiline in the hypophysectomized rat}

Hypophysectomy was performed through a parapharyngeal approach in 15 rats about one hour prior to the experiment. Eight hypophysectomized rats were injected with saline as control in a course of ACTH infusion, and the other 7 with methylenedianiline. In all experiments, ACTH infusion and blood collection were started simultaneously.

The corticosterone secretion rate following infusion of ACTH increased markedly and reached its maximum level 30 minutes after the beginning of ACTH infusion. Thereafter, in control animals injected with saline, a definite change in the corticosterone secretion rate was not observed. On the other hand, in the animals treated with methylenedianiline, the corticosterone secretion rate was markedly depressed.

In control experiments, adrenal blood flow increased gradually by ACTH infusion and reached the maximum level 30 minutes after the start of ACTH infusion. Thereafter no significant change was observed. In experiments of 
TABLE 2. Effect of 4,4'-methylenedianiline on adrenal corticosterone secretion rate in hypophysectomized rats

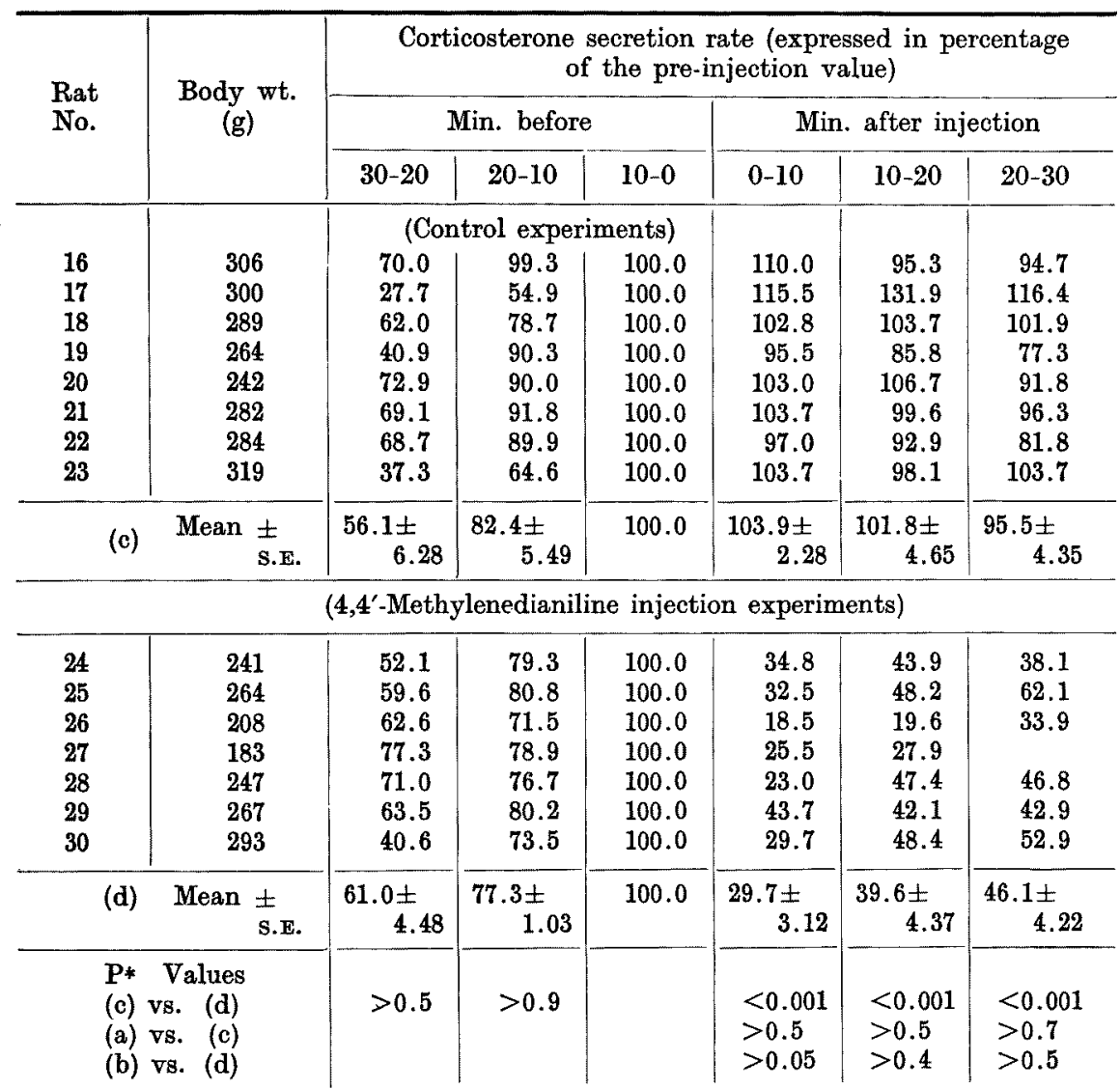

* Probability in statistical analysis by Student's $t$-test

methylenedianiline administration, adrenal blood flow increased at first as observed in the control group; however, administration of methylenedianiline was followed by distinct decrease in adrenal blood flow. By administration of methylenedianiline, respiratory rate increased in hypophysectomized rats as well.

The effect of methylenedianiline or saline (control experiments) on adrenal cortical stimulating effect of ACTH in hypophysectomized rats is illustrated in Fig. 2.

\section{Discussion}

The results of the present study showed that the adrenal corticosterone secretion in response to the surgical stress was markedly decreased by treatment with methylenedianiline. A similar effect of methylenedianiline on 
No. 23 Hypox.

of $319 \mathrm{~g}$

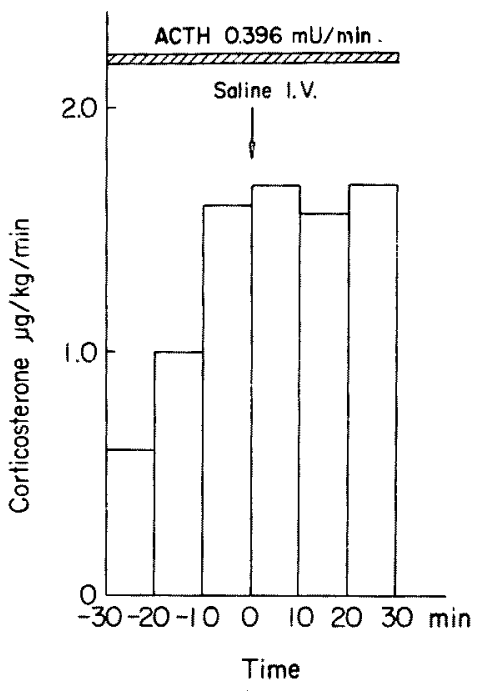

No. 30 Hypox.

\section{of 2939}

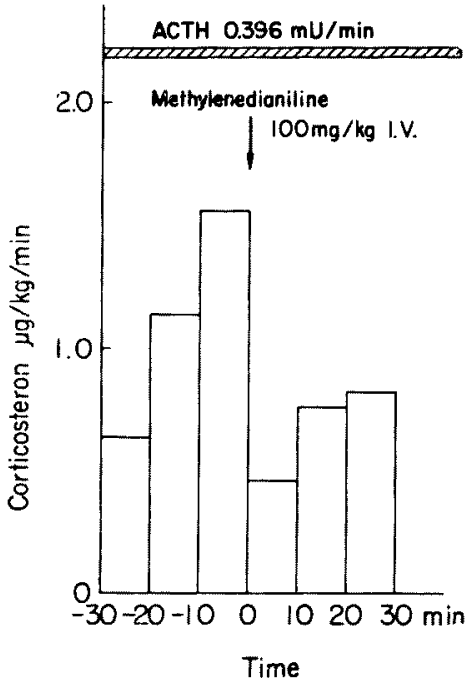

Fig. 2. Effect of 4,4'-methylenedianiline on adrenal corticosterone secretion rate in hypophysectomized, ACTH-infused rats.

corticosterone secretion was observed in hypophysectomized and ACTHinfused rats. Tullner reported that acute intravenous administration of methylenedianiline in a dose of 50 to $100 \mathrm{mg} / \mathrm{kg}$ of body weight produced a definite decrease in 17-hydroxycorticoid output in dogs. By the present investigation it was clarified that the acute intravenous administration of methylenedianiline exhibited the inhibitory effect on the adrenal corticosterone secretion in rats and that the decrease in corticosterone secretion was mainly due to the direct action of methylenedianiline on the adrenal gland.

Tullner ${ }^{7}$ reported also that hypertrophy of the adrenal gland and the uterus was produced by daily oral administration of this compound for a period of 5 to 14 days in castrated female rats, and that this compound elicited progestational proliferation, which was prevented by adrenalectomy as well as by cortisone treatment, in Clauberg rabbit endometrium. Yamashita ${ }^{9}$ showed that a marked increase in carbonic anhydrase of the uterine endometrium could be induced in Clauberg rabbits by treatment with methylenedianiline, and adrenalectomy or cortisone treatment abolished the effect of this compound on uterine carbonic anhydrase activity.

Tullner ${ }^{7}$ and Yamashita ${ }^{9}$ suggested that progestational proliferation of the endometrium or an increase in uterine carbonic anhydrase in rabbits after methylenedianiline treatment might be due to excess secretion of adrenal 
progestogen which was provoked by the blocking effect of methylenedianiline on adrenal steroidogenesis and the increased ACTH secretion of the adenohypophysis induced by lowered circulating corticosterone level. Thus they submitted indirect evidence of an inhibitory effect of methylenedianiline on the corticosterone secretion. The present study showed that corticosterone secretion was depressed directly by methylenedianiline.

Methylenedianiline is a diaminodiphenyl compound which lacks the ketone and methyl groups of amphenone B. Although the exact mechanism of blocking effect of methylenedianiline on the steroidogenesis of the adrenal cortex has not yet been clear, it may be reasonable to assume that the blocking mechanism is the same as that of amphenone $B$, which suppresses the $11 \beta-, 17 \alpha$, and 21-hydroxylation in the adrenal cortex as well as the oxidation of the $\Delta^{5}-3 \beta$-hydroxyl group to the $\Delta^{4}$-3-ketone, as indicated by Rosenfeld and Bascom. ${ }^{2}$

In the present study a decrease in adrenal blood flow was produced by an administration of methylenedianiline in the rat. Vogt ${ }^{4}$ reported that intravenous injection of amphenone $B$ in the rat caused a fall in blood pressure. On the other hand, Tullner? observed an elevation of blood pressure following intravenous administration of methylenedianiline in the dog. $\mathrm{He}$ explained the transient rise in blood pressure to be due to a rapid intravenous injection of of aqueous solution in volumes such as $25-50 \mathrm{ml}$. The transient decrease in adrenal blood flow observed in the present study after injection of methylenedianiline might be due to a fall in blood pressure as described by Vogt $^{4}$ in her study on amphenone $B$.

\section{Acknowledgment}

The author is grateful to Professor T. Suzuki and Dr. K. Yamashita for their suggestion and their help in revising the manuscript and to Drs. K. Hirai, R. Higashi and H. Tanigawa for their valuable assistance in experiments throughout the course of this investigation.

\section{References}

1) Tullner, W.W., Graff, M.M. \& Hertz, R. Amphenone inhibition of adrenal corticosteroid output in the hypophysectomized dog. Endocrinology, 1956, 58, 802-807.

2) Rosenfeld, G. \& Bascom, W.D. The inhibition of steroidogenesis by amphenone B: Studies in vitro with the perfused calf adrenal. J. biol. Chem., 1956, 222, 565-580.

3) Thorn, G.W., Renold, A.E., Goldfien, A., Nelson, D.H., Reddy, W.J. \& Hertz, R. Inhibition of corticosteroid secretion by amphenone in a patient with adrenocortical carcinoma. New Engl. J. Med., 1956, 254, 547-551.

4) Vogt, M. The effects of hexoestrol and of "amphenone B" on morphology and function of the rat adrenal cortex. Yale J. Biol. Med., 1957, 29, 469-479.

5) Peterson, R.E., Hertz, R. \& Lubs, H.A. Suppression of biosynthesis of adrenal cortical steroids in man by amphenone. Proc. Soc. exp. Biol. Med. (N.Y.), 1957, 94, $421-425$.

6) Jenkins, J.S., Meaken, J.W.\& Nelson, D.H. A comparison of the inhibitory effects 
of 2-methyl-1,2-bis (3-pyridyl)-1-propanone and amphenone $\mathbf{B}$ on adrenal cortical secretion in the dog. Endocrinology, 1959, 64, 572-578.

7) Tullner, W.W. Endocrine effects of methylenedianiline in the rat, rabbit and dog. Endocrinology, 1960, 66, 470-474.

8) Silber, R.H., Busch, R.D. \& Oslapase, R. Practical procedure for estimation of corticosterone or hydrocortisone. Clin. Chem., 1958, 4, 278-285.

9) Yamashita, K. Adrenal-dependent effect of 4,4'-methylenedianiline on endometrial carbonic anhydrase. Amer. J. Physiol., 1963, 205, 195-197. 